Revue d'histoire de l'Amérique française

BEVUE D.HISTOIRE DE L'AMÉRIQUE FRANÇAISE

\title{
LATOUCHE, Daniel, À la remorque des transports. Québec, Québec Science Éditeur, 1980, 282 p. Coll. « Les dossiers de Québec Science ».13,50 \$.
}

\section{Georges Massé}

Volume 37, numéro 1, juin 1983

URI : https://id.erudit.org/iderudit/304136ar

DOI : https://doi.org/10.7202/304136ar

Aller au sommaire du numéro

Éditeur(s)

Institut d'histoire de l'Amérique française

ISSN

0035-2357 (imprimé)

1492-1383 (numérique)

Découvrir la revue

Citer ce compte rendu

Massé, G. (1983). Compte rendu de [LATOUCHE, Daniel, À la remorque des

transports. Québec, Québec Science Éditeur, 1980, 282 p. Coll. « Les dossiers de Québec Science ». 13,50 \$.] Revue d'histoire de l'Amérique française, 37(1),

105-109. https://doi.org/10.7202/304136ar d'utilisation que vous pouvez consulter en ligne.

https://apropos.erudit.org/fr/usagers/politique-dutilisation/ 
LATOUCHE, Daniel. A la remorque des transports. Québec, Québec Science Éditeur, 1980, 282 p. (Coll. «Les dossiers de Québec Science»). 13.50\$

Il n'est pas aisé de recenser adéquatement un ouvrage de ce type sans écorcher inutilement et injustement un auteur dont nous apprécions la prolificité, la polyvalence et les dons de communicateur. Il ne convient pas plus de 
manifester un laxisme de mauvais aloi à l'endroit de cette «rencontre de deuxième type» à laquelle nous convient l'auteur et l'éditeur. Une collection de vulgarisation scientifique doit éviter, bien sûr, le Charybde de l'hermétisme et de l'académisme, mais aussi le Scylla de la facilité et de la complaisance. $\mathrm{Si}$ Yanick Villedieu («Demain la santé») et Fernand Seguin («Le sel de la science») évitent ces écueils, il est difficile d'en dire autant de Latouche. Cette étude, qui s'apparente au journalisme scientifique, souffre d'une rédaction hâtive et de l'absence d'une ossature méthodologique pour articuler les idées qui y fourmillent.

L'excuse du dilettantisme ne saurait rescaper un essai que l'on a affranchi trop tôt du chantier. Latouche n'est pas le néophyte que présente, avec humour, la jaquette du volume en prétendant que sa «seule spécialisation en matière de transport» réside dans ses «deux traversées de l'Atlantique en voilier». Sa collaboration au Groupe de Recherches sur le Futur et sa participation, comme auteur et directeur, aux deux volumes de Premier mandat: une prospective à court terme du gouvernement péquiste, l'ont familiarisé avec les problèmes actuels de transport.

Toujours est-il, en ce «39e jour de l'ère post-référendaire» (p. 14), que le mandat, assigné à ce volume, est de fournir «une critique sociale» (p. 11); elle ne se manifeste pourtant qu'aux chapitres 4,5 et 6 , avant de «prendre l'air», au chapitre suivant, et s'estomper au profit d'une analyse politique. Il était plus facile de formuler «a posteriori» qu'il ne s'agit ni «d'une encyclopédie des transports modernes, et encore moins d'un catalogue exhaustif de tous les problèmes qu'on y rencontre» (p. 12), ni d'une recension «d'innovations et de prédictions» (p. 12), que de définir la nature, l'orientation et les paramètres de son «analyse sociologique» (p. 13). Il ne suffit pas de postuler que «c'est surtout à la jonction du politique, de l'économique et du technologique que la bataille se joue» (p. 12) pour valider son intention initiale de privilégier la dimension sociale et politique de la question des transports. Il faut, au contraire, transformer ce postulat en problématique et en faire la démonstration dans le corps du volume. En dehors des chapitres 4 et 5 , où l'enjeu de la «bataille» nous est révélé, le postulat s'étiole et l'intention s'évapore dans les méandres des relations fédérales-provinciales. Même si Latouche nous met en garde contre la «mode» de «l'exagération [que] commande le discours social» (p. 11), lui-même n'y échappe pas dans ses propos introductifs, ni même dans les titres accrocheurs dont il coiffe ses chapitres (L'automobile, coupable ou non coupable?» "Les transports en commun: vite un miracle!», etc.). Exagération surtout dans le traitement qu'il fait de l'histoire.

Il peut sembler paradoxal qu'un historien regimbe à l'assertion que «parler des transports, c'est d'abord parler d'histoire» (p. 13). Et pourtant. L'histoire gargarisante de maints protagonistes du débat pré-référendaire nous a laissé un goût amer. Il y en a encore des relents dans ce bouquin où elle ne sert qu'à confirmer la double hypothèse 1) que les transports «ont tout d'abord joué un rôle déterminant dans le décollage et le développement économique du Québec» (p. 21) et 2) qu'il en résulte, d'une part le retard économique du Québec par rapport à l'Ontario, et d'autre part sa dépendance politique et économique. Force est d'admettre, à la décharge de Latouche, qu'il est victime d'une historiographie qui a presque toujours réduit l'analyse du processus d'industralisation québécois et canadien à l'étude d'un facteur - exogène par 
surcroît - soit celui du rôle de l'Etat, et indirectement du rail. Toutefois, si je ne puis affirmer que l'historiographie ne permet pas de conclure que «...les transports ont créé le fédéralisme canadien, et vice-versa...» (p. 275), il est certain qu'elle n'autorise personne - y compris Latouche - à affirmer que «les transports ont fait du Québec ce qu'il est aujourd'hui» (p. 275). La cause serait donc entendue; un «détour par l'histoire» (p. 17) va permettre à l'auteur de réaliser son oeuvre au noir.

Malheureusement, c'est à un détournement de l'histoire que l'on assiste dans un premier chapitre truffé de clichés. La tentative de lier le passé et le présent n'est pas répréhensible en soi; loin de là. Encore faut-il sentir que le passé n'est pas prisonnier des schématisations du présent; encore faut-il restituer au passé son dynamisme. Cet impératif est complètement évacué par le jeu de saute-mouton qui fait passer le lecteur d'une séquence historique à une autre («voiliers et la marine», au «canot et la fourrure», à «la drave et le bois» et enfin aux «canaux et le blé») en négligeant l'évolution - involution des «staples» et de leur «mode de transport privilégié» (p. 18), et, en figeant l'évolution générale de l'économie. Convenons que la synthèse oblige au survol, au raccourci; mais, il est périlleux de transformer le «détour historique» en vol en rase-mottes. Ce n'est pas ainsi qu'on va exorciser - à supposer que cette dénonciation soit vraie - le «rôle quasi mythique» que nos manuels d'histoire auraient conféré aux transports (p. 18).

Les trop nombreuses imprécisions de ce premier chapitre détournent l'attention des problèmes réels que l'histoire peut nous révéler. Les comparaisons anachroniques aboutissent au même résultat; quel peut être l'apport de cette comparaison voulant que les relations canado-britanniques, antérieures à l'adoption du libre-échange, s'apparentent à une «quasi souveraineté-association avec la mère-patrie» (p. 35)? Il est tout aussi inconvenant que ce détour historique se complaise dans une sorte de syndrome de l'échec, qui nie l'histoire. Echec donc des «Québécois», qui, pour n'avoir «jamais été un peuple de marins tournés vers la mer... ont laissé ce rôle aux colonies de l'Atlantique» (p. 23); et pourtant, prédit-on, «à travers la pêche, les Québécois, auraient pu développer, à la manière des Norvégiens, un intérêt et une compétence pour le transport maritime» (p. 23). Échec encore dans les fourrures. Puis, «avec les canaux et le blé, Montréal voit donc se répéter la vieille histoire du succès initial qui se transforme en échec» (p. 36) avec le rail. À défaut de ne pouvoir ré-écrire l'histoire d'un peuple qui n'aurait, semble-t-il, pas «fait l'histoire» (p. 67, note 3), Latouche l'oblige à se répéter d'échec en échec. Il va jusqu'à «supposer que même si les "Canadiens» avaient été maîtres chez eux, cela n'aurait pas changé grand-chose à cet état de fait» (p. 37). En fin de compte, ces échecs successifs seraient la tare congénitale, imputable au statut colonial originel, d'un peuple que le destin condamne à se laisser phagocyter.

Il est à souhaiter que cette vision pessimiste, téléologique et mythique (origines), soit seulement dictée par le dépit que l'échec du référendum impose momentanément à un intellectuel qui, habituellement, démontre plus de discernement. À preuve, ce constat que la Confédération confirme le «double statut de minorité économique et politique des Canadiens français» (p. 37). Toutefois n'est-ce pas une évaluation légère et expéditive que de faire porter le poids de cette «minorisation» sur les épaules de la génération de politiciens canadiens-français de la période de l'Union? Pourquoi auraient-ils été les seuls 
à ne pas avoir «d'objectifs bien définis» (p. 40)? Latouche sait qu'un «projet politique d'envergure», c'est-à-dire l'indépendance nationale, «n'était plus envisageable depuis 1837» (p. 41). Si la politique est parfois l'art du possible, pourquoi, dans ce contexte, stigmatiser l'absence de "projet collectif» des députés francophones et réduire l'Assemblée législative du Canada-Uni à un «vaste théâtre» peuplé de marionnettes canadiennes-françaises que les Anglais manipulent au gré de leur «nouvelle mise en scène» (p. 39)? L'auteur devrait savoir que «Le grand jeu de la politique» (chapitre 2) est plus complexe que ses propos dérisoires veulent bien laisser croire.

Le «détour» historique aboutit enfin au «coup de l'Intercolonial» (p. 5158 ) et à la question de la «Confédération et les transports» (p. 58-61). Relativement bien articulés, ces propos veulent surtout démontrer: primo, que les hommes politiques canadiens ne s'intéressent à l'Intercolonial qu'à partir du moment où l'on discute d'une union des colonies atlantiques, et secundo, que l'A.A.N.B. en vertu de l'attribution des pouvoirs législatifs et financiers, fait du gouvernement central le maître d'oeuvre d'un développement économique dont les transports seront le fer de lance.

Le troisième chapitre traite de la "géographie économique des transports» (p. 63-83); il vient chapeauter toute la première section du volume. Il est dommage que Latouche, au lieu de s'attarder à la détection des «contraintes de l'espace québécois» (66 ss), n'ait pas conféré une portée méthodologique à cette géographie économique; s'il lui avait assigné un rôle de poteau de signalisation, pour guider le lecteur dans son détour historique, il aurait peut-être réussi à justifier les libertés qu'il prend dans le traitement de l'histoire. Plutôt que de croire que «réfléchir sur les transports est une tâche ingrate...» (p. 278), il eût fallu qu'il étaye l'affirmation que le transport est une activité économique à «caractère structurant» (p. 63). Il fallait démontrer, souligner tout au moins, 1) l'articulation ou la non-articulation des réseaux en un système de transport dynamique; 2) l'adéquation ou la non-adéquation de celui-ci par rapport au système économique qu'il dessert et 3 ) au domaine politique qu'il sous-tend.

Il est probablement discriminatoire qu'une fois sorti de ce détour cahoteux par les chemins de l'histoire, lequel ne représente qu'environ le tiers du volume, il faille accélérer pour mieux nous conformer aux contraintes de ce compte rendu. Donc, retour au sujet principal que l'auteur n'aurait jamais dû quitter. En effet, ses considérations sur l'actualité, mieux sur l'histoire immédiate, sont indéniablement plus pertinentes et cohérentes. Si ces qualités confèrent un intérêt certain à toute la seconde section de l'ouvrage, c'est tout simplement dû au fait que l'auteur connaît mieux les questions qu'il y aborde; c'est parce qu'il peut donner libre cours à son imagination et jeter un regard critique, et non un «regard plus froid» (p. 79) sur les problèmes de transport, tant ceux du réseau aérien (chapitre 8) que ceux du réseau fluvial (chapitre 8) et ferroviaire (chapitre 9), qui confrontent la société québécoise actuelle.

En premier chef, se pose la question de la place de l'automobile dans les villes modernes (chapitre 4). Le procureur Latouche montre emphatiquement que les arguments de ceux qui l'accusent de détruire la ville reposent sur une vision idyllique de la ville ancienne (p. 89), et qu'il suffirait de quelques corrections pour régler l'embouteillage urbain (p. 90). L'apport principal de ce plaidoyer réside dans la récusation de la perspective manichéenne dans laquelle 
s'enferment trop souvent les détracteurs de l'automobile. Il les invite donc à faire preuve de réalisme politique s'ils veulent convaincre les citadins des avantages indéniables (p. 143 ss) du transport public (chapitre 5).

Il va sans dire que le «mal urbain» (p. 159) déborde largement la question des transports; c'est par le recours à l'imagination que l'auteur prétend, un peu naïvement, qu'on pourra éventuellement le régler. À ce chapitre de «l'imagination au pouvoir» (chapitre 6), s'inspirant de plusieurs exemples et études européennes, il présente les trois volets d'une alternative à la circulation automobile: la marche à pied (rue piétonnière), la bicyclette et le tramway. Une combinaison et une intégration judicieuse de ces trois éléments solutionneraient les problèmes du transport urbain. 